\title{
Radar-Based Health Monitoring
}

\author{
Dominique Schreurs ${ }^{*}$, Marco Mercuri ${ }^{*}$, Ping Jack Soh ${ }^{*}, \dagger$, Guy Vandenbosch ${ }^{*}$ \\ *KU Leuven, Div. ESAT-TELEMIC, Leuven, Belgium \\ Email: [dominique.schreurs, marco.mercuri, pingjack.soh, guy.vandenbosch]@esat.kuleuven.be \\ ${ }^{\dagger}$ Universiti Malaysia Perlis, School of Computer \& Communication Engineering, Perlis, Malaysia
}

\begin{abstract}
Radar techniques aiming at health monitoring have been investigated in recent years. The possibility of contactless monitoring human individuals has aroused interest in many applications especially where the use of wearable sensors generates discomfort which not only may affect the results but also imposes a risk factor. Radar technologies represent therefore the new emerging solution to promote the well-being of elderly people both in home and clinical environments. This paper provides an overview on recent advances in health monitoring using radar techniques. Moreover, an example of a concrete health monitoring system is also reported.

Index Terms - Doppler, fall detection, health monitoring, radar remote sensing, SFCW radar, UWB-IR radar, vital signs.
\end{abstract}

\section{INTRODUCTION}

Wireless technologies have been recently investigated as an emerging key for health monitoring to provide benefits of diagnosis, treatment, and detection of emergency situations in home and clinical environments.

The most developed approach are the wireless body area networks (WBANs) which are predicted to proliferate in healthcare in the next years. They involve sensors attached to the human body that transmit continuously data to physicians or caregivers. Devices integrating sensors to record biomedical signals such as heart pattern (ECG), blood oxygenation saturation $\left(\mathrm{SpO}_{2}\right)$, brain activity (EEG), blood pressure (BP), and motion detectors, such as accelerometers, gyroscopes, and tilt sensors, have been developed or are under investigation. The main problem with these developments is that they produce discomfort and elderly people tend to forget wearing them.

For that reason, in recent years, remote health monitoring has become an increasingly hot research topic worldwide. The monitoring of human beings can be made remotely and therefore contactless by adopting radar techniques. In fact, the mechanical movements of heart, chest (lungs), and body cause changes in frequency, phase, and arrival time of the wave reflected from the person, and in this way heartbeat, respiration rate, and motion speed can be measured.

This paper provides an overview on the main radar architectures currently used in contactless health monitoring (Section II). Moreover, an example of a practical implementation is presented in Section III. It concerns a radar sensor based system aiming at contactless fall detection, tagless localization, and human gait classification. Finally, conclusions are drawn in Section IV.

\section{RADAR ARCHITECTURES FOR HEALTH MONITORING}

\section{A. Radar Techniques}

Various radar architectures have been investigated, based on frequency, phase, and arrival time changes.

A pure Continuous Wave (CW) radar, or Doppler radar, transmits an RF single-tone continuous-wave signal, which is reflected off a target and then demodulated in the receiver. By the Doppler effect, a radio wave reflected at the moving surface undergoes a frequency shift proportional to the surface velocity, and therefore it is possible to measure the target's speed without ambiguity. If the surface has a periodic motion, like that of the heart and chest, this can also be seen as a phase shift proportional to the surface displacement. If the displacement is small compared to the wavelength, a circuit that couples both the transmitted and reflected signals to a mixing element can produce an output signal with a low frequency component that is directly proportional to the object displacement. Demodulating the phase will then give a signal proportional to the chest-wall position that contains information about movement due to heartbeat and respiration, making the CW radar the most straightforward architecture for vital signs monitoring.

However, the main limitation of a Doppler radar is that it is not able to determine absolute distances. Opposite to this, ultra-wideband (UWB) radars can provide both speed and absolute distance of a target. Moreover, they can resolve closely spaced targets in range, providing high-resolution range profiles and increasing the signal-to-clutter ratio so that targets become detectable above clutter and noise. UWB radars for health monitoring are mainly based on UWB-IR (Impulse-Radio) radar and stepped-frequency continuous wave (SFCW) radar. The range resolution for both categories is determined by the formula:

$$
\Delta R=\frac{C}{2 B}
$$

where $c$ is the speed of light and $B$ is the total bandwidth. The latter is associated with the shape of the waveform and it is achieved in a different way for each category.

An UWB-IR radar, also known as time-domain radar, operates by sending short electromagnetic pulses with a fixed pulse repetition frequency (PRF) and by receiving the echoes reflected by the target. The time delay between the 
transmission of the pulse and the reception of the echo is proportional to the distance from the target to the radar. The radar transmitter generates a sequence of short pulses with leading-edge duration in the order of tens of picoseconds. This pulse sequence is reflected from the object and collected by the receiver. As the emitted wave is reflected by a target, the PRF of the reflected wave is modulated by the target's movement, so this change can be used to monitor a person. The narrower are the pulses, the more accurate is the measurement of the range, at the expense of a broader transmitted spectrum.

Alternatively, an SFCW radar, also known as frequencydomain radar, transmits and receives continuously a train of $N$ CW pulses whose frequencies are increased from pulse to pulse by a fixed frequency step $\Delta f$. The range information is proportional to the difference in frequency between the transmitted and the reflected signal. The larger is the frequency deviation in a given interval, the more accurate is the range resolution. The high resolution range measurement is achieved by processing the echoes of the $N \mathrm{CW}$ pulses. These pulses have a narrow bandwidth, making the instantaneous radar bandwidth narrow resulting in higher signal-to-noise ratio (SNR) and radar sensitivity. Moreover, it implies that the hardware requirements in terms of analog-todigital-converters (ADCs) and processors become less stringent.

\section{B. Applications in Health Monitoring}

Remote health monitoring using radar techniques can be beneficial for several potential applications in health care both in home and clinical environments.

Doppler radars have been investigated for precise detection of cardiopulmonary activity [1]. Moreover, they represent a tool for Sudden Infant Death Syndrome (SIDS) and sleep apnea monitoring [2], and in tracking accurately and systematically irregular heart or breathing patterns. They have been also validated as potential substitute of conventional respiratory devices in clinical environments [3].

UWB-IR radars have been also used in vital signs detection [4]. Moreover, impulse signals are also useful to penetrate solid media such as rocks, building walls, etc, making this technology suitable for applications such as people rescue missions after for instance an earthquake or building collapse [5]. The main difficulty is to detect and to separate the weak cardiopulmonary motion response of a trapped victim from other non-stationary movements originating by moving objects or people in the scene of interest.

SFCW radars have been investigated for noncontact fall detection, tagless localization, and vital signs monitoring in the home environment [6]. Moreover, they have been also explored for contactless breast cancer detection in clinical environments [7].

\section{HEALTH MONITORING SYSTEM}

In this Section an example of a health monitoring system designed for contactless fall detection, human gait classification, and tagless localization is described. It combines the features of the radar architectures described in the previous Section. The system consists of a sensor and a base station, combining radar sensing, wireless communications, and data processing techniques (Fig. 1) [8]. The sensor integrates a radar module, a Zigbee chip, and a microcontroller. The radar generates continuously a waveform that is based on a hybrid approach where a single tone at 5.8 $\mathrm{GHz}$ is transmitted for $T_{D}=1 \mathrm{~s}$, and then alternated with a SFCW signal of $2 \mathrm{~ms}$ (Fig. 1) [9]. The latter consists of $N=40$ CW pulses of $T=50 \mu$ s each, positioned in the UWB band and starting from $6 \mathrm{GHz}$ with a fixed frequency increment of $\Delta f=25 \mathrm{MHz}$. This involves a total bandwidth $B=\Delta f N$ of 1 $\mathrm{GHz}$ allowing a range resolution $\Delta R$ of $15 \mathrm{~cm}$. This solution allows to detect continuously the speed of a target and its absolute distance every second, while keeping the complexity of the sensor simple and, at the same time, satisfying the European and Federal Communications Commission (FCC) UWB mask requirements. This is in contrast to the traditional UWB technologies where the dwell time of the pulses should be extremely reduced to satisfy the UWB spectrum mask and to have a proper transmit power level, thus increasing data acquisition rates and therefore hardware complexity. The baseband signals are subsequently digitized and transmitted wirelessly by Zigbee to a base station for data processing.

The baseband speed signals can then be analyzed using machine learning techniques to distinguish a fall incident from normal movements (e.g., walking, sitting down, standing up) [10]. The key is to detect the changes in speed of a person during a fall or a normal movement. Fig. 2 and Fig. 3 show the speed signals during a fall event and walking, resp. The frequency of the signals is proportional to the radial velocity of the person during the movements. During the fall, the speed increases over time, while it is quite constant during a walk. In addition, a similar classification technique can be also used to distinguish different subjects detecting the differences in speed in their way of walking, as shown in Fig. 3.

On the other hand, the SFCW baseband samples are used to determine the target's absolute distance [11]. Fig. 4 shows the range profile of a person standing at $3 \mathrm{~m}$ away from the radar sensor.

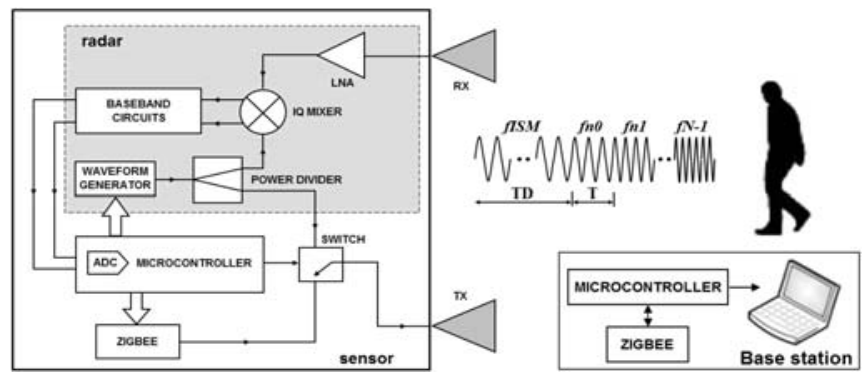

Fig. 1. Block diagram of the health monitoring system. 


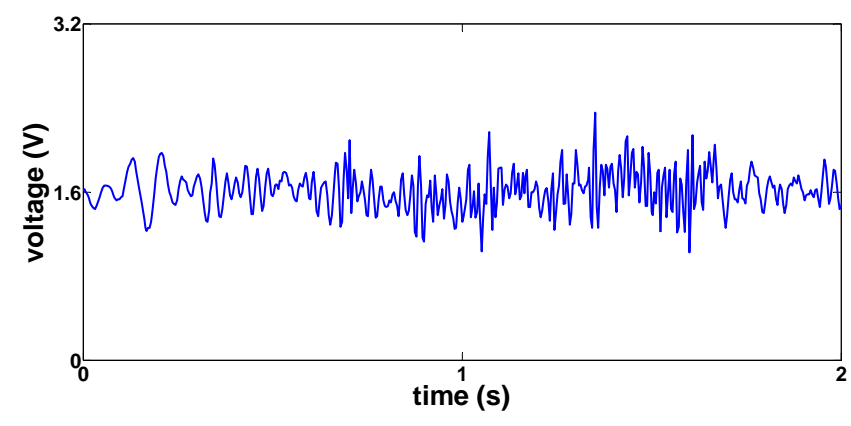

Fig. 2. Speed signal during a fall incident.

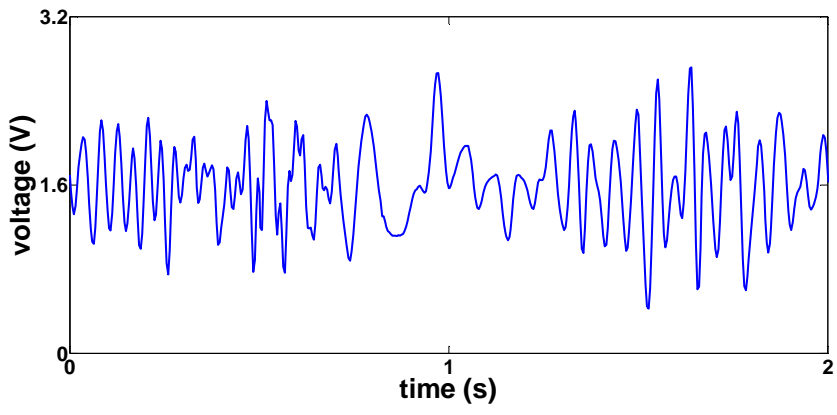

(a)



(b)

Fig. 3. Speed signals during a walking movement of subject A (a) and of subject B (b). The two individuals are walking the same path and the resulting signals show a clear difference in speed. Subject B in fact walks faster than Subject A.

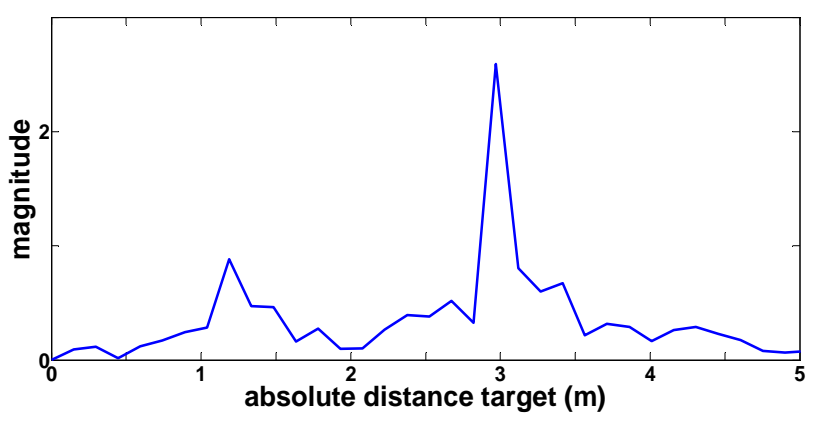

Fig. 4. Range profile of a subject at $3 \mathrm{~m}$ away from the sensor.

\section{CONCLUSION}

Radar technologies have the advantage of high detection sensitivity and high range resolution. This makes them the most suitable approach for noncontact health monitoring in both home and clinical environments. This paper described and analyzed the different approaches adopted in such biomedical radar systems. Moreover, a practical system for fall detection, tagless localization, and human gait classification has also been presented.

\section{ACKNOWLEDGEMENT}

This work was supported by FWO-Flanders, KU Leuven GOA and IOF leverage projects, and the Hercules Foundation.

\section{REFERENCES}

[1] A.D. Droitcour, V.M. Lubecke, J. Lin, and O. Boric-Lubecke, "A microwave radio for Doppler radar sensing of vital signs," IEEE MTT-S International Microwave Symposium, vol. 1, pp. 175-178, June 2001.

[2] C. Li, J. Lin, and Y. Xiao, "Robust overnight monitoring of human vital signs by a non-contact respiration and heartbeat detector," 28th Annu. IEEE Int. Eng. Med. Biol. Soc. Conf., pp. 2235-2238, Aug. 2006.

[3] A. D. Droitcour et al., "Non-contact respiratory rate measurement validation for hospitalized patients," 31st Annu. IEEE Int. Eng. Med. Biol. Soc. Conf., pp. 53-56, Sep. 2009.

[4] I.Y. Immoreev, S. Samkov, and T.-H. Tao, "Short-distance ultra wideband radars," IEEE Aerospace and Electronic Systems Magazine, vol. 20, no. 6, pp. 9-14, June 2005.

[5] G. Ossberger, T. Buchegger, E. Schimback, A. Stelzer, and R. Weigel, "Non-invasive respiratory movement detection and monitoring of hidden humans using ultra wideband pulse radar," Conference on Ultrawideband Systems and Technologies, pp. 395-399, May 2004.

[6] M. Mercuri, D. Schreurs, and P. Leroux, "SFCW microwave radar for in-door fall detection,” IEEE Topical Conf. Biomedical Wireless Technologies (BioWireless), pp. 53-56, Jan. 2012.

[7] M. Bassi, A. Bevilacqua, A. Gerosa, and A. Neviani, "Integrated SFCW transceiver for UWB breast cancer imaging: architectures and circuit constraints," IEEE Trans. Circuits Sys., vol. 59, no. 6, pp. 1228-1241, 2012.

[8] M. Mercuri, P. J. Soh, G. Pandey, P. Karsmakers, G. A. E. Vandenbosch, P. Leroux, and D. Schreurs, "Analysis of an indoor biomedical radar-based system for health monitoring," IEEE Trans. Microwave Theory Tech., vol. 61, no. 5, pp. 20612068, 2013.

[9] M. Mercuri, D. Schreurs, and P. Leroux, "Optimised waveform design for radar sensor aimed at contactless health monitoring," IET Electronic Letters, vol. 48, no. 20, pp. 1255-1257, 2012.

[10] P. Karsmakers, T. Croonenborghs, M. Mercuri, D. Schreurs, and P. Leroux, "Automatic in-door fall detection based on microwave radar measurements," European Radar Conference (EuRAD), pp. 202-205, Nov. 2012.

[11] M. Mercuri, P. J. Soh, D. Schreurs, and P. Leroux, "A practical distance measurement improvement technique for a SFCWbased health-monitoring radar in real indoor environment," Automatic RF Techniques Group Conference (ARFTG), pp. 1-4, June 2013. 